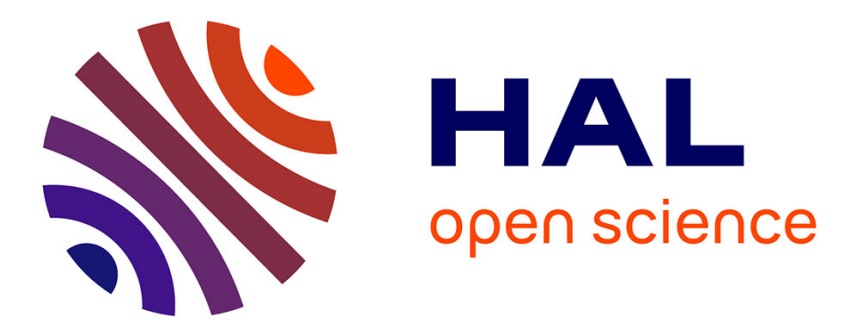

\title{
Hard X-ray Photon-in Photon-out Spectroscopy as a Probe of the Temperature-Induced Delocalization of Electrons in Nanoscale Semiconductors
}

\author{
Ofer Hirsch, Kristina Kvashnina, Christoph Willa, Dorota Koziej
}

\section{To cite this version:}

Ofer Hirsch, Kristina Kvashnina, Christoph Willa, Dorota Koziej. Hard X-ray Photon-in Photon-out Spectroscopy as a Probe of the Temperature-Induced Delocalization of Electrons in Nanoscale Semiconductors. Chemistry of Materials, 2017, 29 (4), pp.1461-1466. 10.1021/acs.chemmater.6b05218 . hal-01691847

\section{HAL Id: hal-01691847 https://hal.science/hal-01691847}

Submitted on 24 Jan 2018

HAL is a multi-disciplinary open access archive for the deposit and dissemination of scientific research documents, whether they are published or not. The documents may come from teaching and research institutions in France or abroad, or from public or private research centers.
L'archive ouverte pluridisciplinaire $\mathbf{H A L}$, est destinée au dépôt et à la diffusion de documents scientifiques de niveau recherche, publiés ou non, émanant des établissements d'enseignement et de recherche français ou étrangers, des laboratoires publics ou privés. 


\title{
Hard X-ray Photon-in Photon-out Spectroscopy as a Probe of the Temperature-Induced Delocalization of Electrons in Nanoscale Semiconductors
}

\author{
Ofer Hirsch, ${ }^{\dagger}$ Kristina Kvashnina, ${ }^{\ddagger}, \|$ Christoph Willa, ${ }^{\dagger}$ and Dorota Koziej ${ }^{*},{ }^{\dagger}$ \\ ${ }^{\dagger}$ Laboratory for Multifunctional Materials, Department of Materials, ETH Zürich, Vladimir-Prelog-Weg 5, 8093 Zurich, Switzerland \\ "The European Synchrotron Research Facility, CS40220, 38043 Grenoble Cedex 9, France \\ "Institute of Resource Ecology, Helmholtz-Zentrum Dresden-Rossendorf, P.O. Box 510119, 01314 Dresden, Germany
}

\section{Supporting Information}

ABSTRACT: Hard X-ray photon-in photon-out spectroscopy has so far mainly been applied to investigate fundamental physical phenomena in superconductors and chemical reactivity of bioinorganic, photocatalytic, and catalytic materials. Here, we show, with the example of $\operatorname{Pr}_{6} \mathrm{O}_{11}$ nanoparticles, an n-type semiconductor, how high-energy resolution fluorescence detected (HERFD) X-ray absorption near edge structure (XANES) can be used to track the changes of partially filled $f$-bands. We observe a reversible variation of the spectral features related to the tetravalent Pr ions upon heating and cooling, whereas structural and chemical transformations can be excluded. We assign these changes to the occupancy of the $\mathrm{O} 2 p-\operatorname{Pr} 4 f$ band and show that they

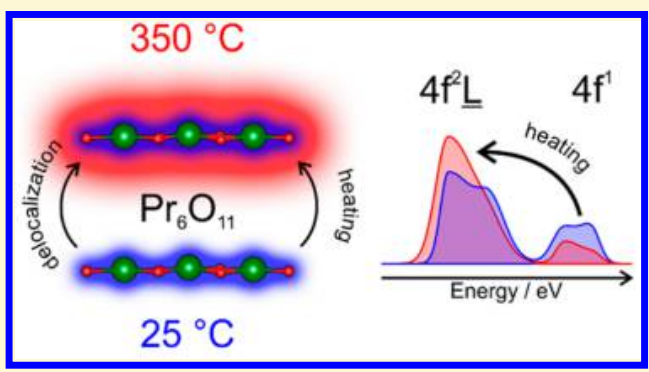
directly relate to changes in the electrical conductance. Our results demonstrate how HERFD-XANES can be used to particularly study in situ the electronic properties of $f$-electrons in a semiconductor and how this method can be further extended to other classes of semiconducting nanomaterials.

\section{INTRODUCTION}

Semiconducting nanoparticles are the hub of many emerging applications ranging from thermoelectrics ${ }^{1}$ to a variety of photocatalytic ${ }^{2}$ and electronic devices. ${ }^{3-6}$ Different techniques allow characterizing the electrical conductivity of nanomaterials, but most of them impose very strict requirements for the sample preparation, for example, the geometry and the properties of the electrical contacts. For nanomaterials, we still grapple with the overarching picture of different length scales, from the macroscopic electrical properties to the electronic states participating in the charge transfer. Particularly, accounting for the density of state and related parameters like metal-oxygen hybridization, delocalized charge carriers with respect to the elemental composition and electronic and crystal structure of the nanoparticle at ambient conditions is still very challenging.

Hard X-ray photon-in photon-out spectroscopy is elementand structure-sensitive and has been shown to probe changes in the density of unoccupied states of rare-earth materials during chemical reactions. ${ }^{7-10}$ The pre-edge region probes the $f$-states directly via $2 p \rightarrow 4 f$ quadrupole transitions and a full RIXS map gives further insights into the exact nature of those states. ${ }^{11}$ Whereas, in the high-energy resolution fluorescence detected X-ray absorption near edge structure (HERFD-XANES) spectrum, shape and position of edge and number, position, and intensity of the features in the postedge resolve the correlation between RE valence and $f$-electron occupancy. ${ }^{8,11}$
Here, we demonstrate that hard X-ray photon-in photon-out spectroscopy can be used as a contactless probe to track the temperature-induced changes of the delocalization of $f$ electrons, which are taking part in the electrical conductivity. To this end, we focus on $\operatorname{Pr}_{6} \mathrm{O}_{11}$ because it is an $\mathrm{n}$-type semiconductor exhibiting rich spectroscopic features related to $f$-electrons in the $\operatorname{Pr} \mathrm{L}_{3}$-edge HERFD-XANES and RIXS spectra. The conduction mechanism in $\operatorname{Pr}_{6} \mathrm{O}_{11}$ is described with the small polaron model, where the dimensions of the polarons are of the same size as the distance between two sites in the lattice. $^{12}$ The $f$-states of $\operatorname{Pr}$ and the $\mathrm{O} 2 p$-states overlap, hybridize, and form a band. ${ }^{13}$ The local coordination and hence the crystal structure determines the degree of hybridization and the filling level of this band. We calculate the RIXS maps of tri-, tetra-, and mixed-valent $\operatorname{Pr}$ and correlate them with the measured HERFD-XANES spectra during temperature-induced transformation of $\operatorname{Pr}(\mathrm{OH})_{3}$ to $\operatorname{Pr}_{6} \mathrm{O}_{11}$. Surprisingly, we observe strong discrepancies between spectra of $\mathrm{Pr}_{6} \mathrm{O}_{11}$ measured at room and at high temperatures, whereas the in situ PXRD and TGA remain unchanged. Thus, we can exclude the chemical and structural transformation. Upon repeated heating and cooling of the $\operatorname{Pr}_{6} \mathrm{O}_{11}$ nanoparticles, we observe a reversible variation of the spectral features related to the tetravalent $\mathrm{Pr}$ ions. We can unambiguously assign these changes to the

Received: December 9, 2016

Revised: January 24, 2017

Published: February 12, 2017 
occupancy of the $\mathrm{O} 2 p-\operatorname{Pr} 4 f$ band and show that they directly relate to changes in the electrical conductance.

\section{EXPERIMENTAL SECTION AND CALCULATION METHODS}

Nanoparticle Synthesis. The synthesis of $\operatorname{Pr}(\mathrm{OH})_{3}$ nanoparticles is analogous to the previously reported synthesis of $\mathrm{La}(\mathrm{OH})_{3}{ }^{7}$ All chemicals were stored and handled in an oxygen- and water-free atmosphere in a glovebox and used as purchased without further purification. Acetophenone (4.68 mL, $40 \mathrm{mmol}, 99.0 \%$ Fluka) was added to $\operatorname{Pr}\left(\mathrm{O}^{i} \mathrm{Pr}\right)$ ( $159.09 \mathrm{mg}, 0.5 \mathrm{mmol}$, 99.9\% Strem Chemicals) in a reaction vessel containing a stirring magnet. The vessel was sealed with a Teflon cap, and the reaction was carried out in a CEM Discovery microwave reactor at $200{ }^{\circ} \mathrm{C}$ for $20 \mathrm{~min}$. The synthesized particles were separated from the reaction liquid by centrifugation, washed twice with ethanol and once with acetone, and dried at $60{ }^{\circ} \mathrm{C}$ for $12 \mathrm{~h}$. To determine the temperature at which the phase transformation from $\operatorname{Pr}(\mathrm{OH})_{3}$ to $\mathrm{Pr}_{6} \mathrm{O}_{11}$ is fully completed, we measured in situ PXRD (Supporting Information Figure S1). $\operatorname{Pr}_{6} \mathrm{O}_{11}$ nanoparticles, used here as a reference, were obtained by heating the $\operatorname{Pr}(\mathrm{OH})_{3}$ powder to $500{ }^{\circ} \mathrm{C}$ for $2 \mathrm{~h}$ with a heating rate of $5{ }^{\circ} \mathrm{C} / \mathrm{min}$.

X-ray Spectroscopy. HERFD-XANES experiments were carried out at ID26 at the European Synchrotron Research Facility in Grenoble, France. The incident beam energy was set to the $\mathrm{Pr} \mathrm{L}_{3}$ edge at $5964 \mathrm{eV}$ selected with a double Si (111) monochromator. The spectrometer in Rowland geometry was tuned to the maximum of the $\operatorname{Pr} \mathrm{L} \beta_{2}$ emission line at $5849.63 \mathrm{eV}$ with three spherically bent $\mathrm{Ge}$ (333) crystals at a Bragg angle of $77^{\circ}$. The total energy resolution was $0.85 \mathrm{eV}$. A typical HERFD-XANES scan was measured from 5945 to $6050 \mathrm{eV}$ with a step size of $0.1 \mathrm{eV}$ and took $300 \mathrm{~s}$ per scan. The RIXS maps were measured from 5954 to $5964 \mathrm{eV}$ in the incident energy range and from 5840 to $5857 \mathrm{eV}$ in the emission energy range with step sizes of 0.1 and $0.4 \mathrm{eV}$, respectively. The ex situ measured reference samples were pressed into pellets of $2.5 \mathrm{mg}$ of $\operatorname{Pr}(\mathrm{OH})_{3}$ or $\operatorname{Pr}_{6} \mathrm{O}_{11}$ nanoparticles and $20 \mathrm{mg}$ of boron nitride. For the in situ heating experiments, a capillary was filled with $\operatorname{Pr}(\mathrm{OH})_{3}$ and placed in a custom-made furnace from the ESRF sample environmental pool and heated to $800{ }^{\circ} \mathrm{C}$ with a heating rate of $5{ }^{\circ} \mathrm{C} / \mathrm{min}$. HERFD-XANES was continuously recorded, $300 \mathrm{~s}$ per scan.

Valence-to-core (vtc) X-ray emission spectroscopy (XES) was measured on the same spectrometer equipped with four spherically bent $\mathrm{Ge}(333)$ crystals at a Bragg angle of $73^{\circ}$. The incident beam energy was fixed at $5976.5 \mathrm{eV}$, and the emission was measured from 5890 to $5971.5 \mathrm{eV}$ with a step size of $0.3 \mathrm{eV}$.

Multiplet Calculations. The multiplet calculations were performed with the MissingCode program ${ }^{14,15}$ for $\operatorname{Pr}^{3+}$ and $\operatorname{Pr}^{4+}$ ions. First, the absorption process of the $2 p \rightarrow 4 f$ quadrupole transitions was calculated, followed by the emission resulting from a $4 d \rightarrow 2 p$ dipole transition. The considered electron configurations were $2 p^{6} 4 d^{10} 4 f^{\mathrm{N}} \rightarrow 2 p^{5} 4 d^{10} 4 f^{\mathrm{N}+1} \rightarrow$ $2 p^{6} 4 d^{9} 4 f^{\mathrm{N}+1}$. The intensities of the different final states were calculated with the Kramers-Heisenberg equation, ${ }^{11,16}$ including a core-hole lifetime broadening of the $2 p$ of $3 \mathrm{eV}$ and of the $4 d$ hole of $0.5 \mathrm{eV}$ with an instrumental broadening of $0.3 \mathrm{eV}$ for the incident beam and $0.6 \mathrm{eV}$ for the analyzer crystals. The calculations took $4 f-4 f, 2 p-4 f$, and $4 d-4 f$ interactions as well as the spin-orbit coupling of the open shells into account. The values of the Slater integrals were taken from the Cowan code and were scaled to $80 \%$ for the different interactions. The spin-orbit coupling was not scaled. The values of the reduced Slater integrals are listed in Table S1, Supporting Information.

Evaluation of RIXS Maps and In Situ Data. The RIXS maps were calculated on the basis of multiplet theory. The multiplet calculations were performed with the MissingCode program ${ }^{14,15}$ for $\mathrm{Pr}^{3+}$ and $\operatorname{Pr}^{4+}$ ions, where we calculated first the quadrupole absorption process, followed by the emitting dipole transition. The final state intensities were calculated with the Kramers-Heisenberg equation, ${ }^{11,16}$ including a core-hole lifetime broadening at the intermediate $(2 p$ hole) and final ( $4 d$ hole) state and additional broadening due to the incident beam width and resolution of the analyzer crystals. The Slater integral values for the different interactions were taken from the Cowan code and scaled to $80 \%$. All reduced values are listed in Table S1, Supporting Information.

The in situ HERFD-XANES data were treated with the fityk program (Version 0.9.8). ${ }^{17}$ All normalized spectra were first fitted with an arctangent step function, and the spectral features were fitted with SplitGaussians. Four such functions were fitted into the $\operatorname{Pr}_{6} \mathrm{O}_{11}$ HERFD-XANES spectra, and the energy positions of the maxima were saved. Afterward, the in situ spectra were fitted with two, three, or four SplitGaussians (see text) with their maxima fixed to the ex situ determined values. The areas of the fitted Gaussians were used to determine the $f$ electron occupancy, $n_{f}{ }^{8,18,19}$ with eq 1 .

$$
n_{f}=1+\frac{A\left(f^{2}\right)+A\left(f^{2} \underline{L}\right)}{A_{e g}\left(f^{1}\right)+A_{t 2 g}\left(f^{1}\right)+A\left(f^{2}\right)+A\left(f^{2} \underline{L}\right)}
$$

where $A\left(f_{n}\right)$ corresponds to the area of the fitted SplitGaussians of the respective feature.

The in situ pre-edge analyses were carried out with Matlab's trapz-function to obtain the area under the pre-edge up to an energy of $5960 \mathrm{eV}$ (dashed line in Figure 2c). We normalized the area by the area of the spectra measured at $25^{\circ} \mathrm{C}$,

$$
n_{A}=1-\frac{A}{A\left(25^{\circ} \mathrm{C}\right)}
$$

where $A$ is the area below the pre-edge at a given temperature and $A\left(25^{\circ} \mathrm{C}\right)$ the pre-edge area for the first in situ measurement of $\operatorname{Pr}(\mathrm{OH})_{3}$ at $25{ }^{\circ} \mathrm{C}$.

PXRD. In situ PXRD data was collected on a Panalytical Empyrean diffractometer equipped with a PIXCEL1D detector using $\mathrm{Cu} \mathrm{K} \alpha$ radiation. The $\operatorname{Pr}(\mathrm{OH})_{3}$ powder was prepared on an $\mathrm{Al}_{2} \mathrm{O}_{3}$ sample holder and mounted in an AP HTK-1200N oven. The incident beam optics consisted of 0.04 Soller slits, a divergence slit of $1 / 4^{\circ}$, and an antiscatter slit of $1 / 2^{\circ}$. On the diffracted beam side, 0.04 Soller slits, a Ni-filter, and a $1 / 4^{\circ}$ divergence slit were used. Additionally, high-resolution synchrotron PXRD data was collected at the Swiss-Norwegian Beamline at the ESRF with a Dectris Pilatus2M detector and an incident energy of $17780.61 \mathrm{eV}$ corresponding to a wavelength of $0.69730 \AA$. The nanoparticles were filled into a quartz capillary, which was mounted on a goniometer head and rotated. The acquisition time was $60 \mathrm{~s}$ per sample. Rietveld refinement was performed using the FullProfSuite software. ${ }^{20,21}$ The background was defined as a linear interpolation of background points with refineable height and the peak shape function used to fit the patterns was the pseudo-Voigt function with axial divergence asymmetry. The analysis of the $\operatorname{Pr}_{6} \mathrm{O}_{11}$ 


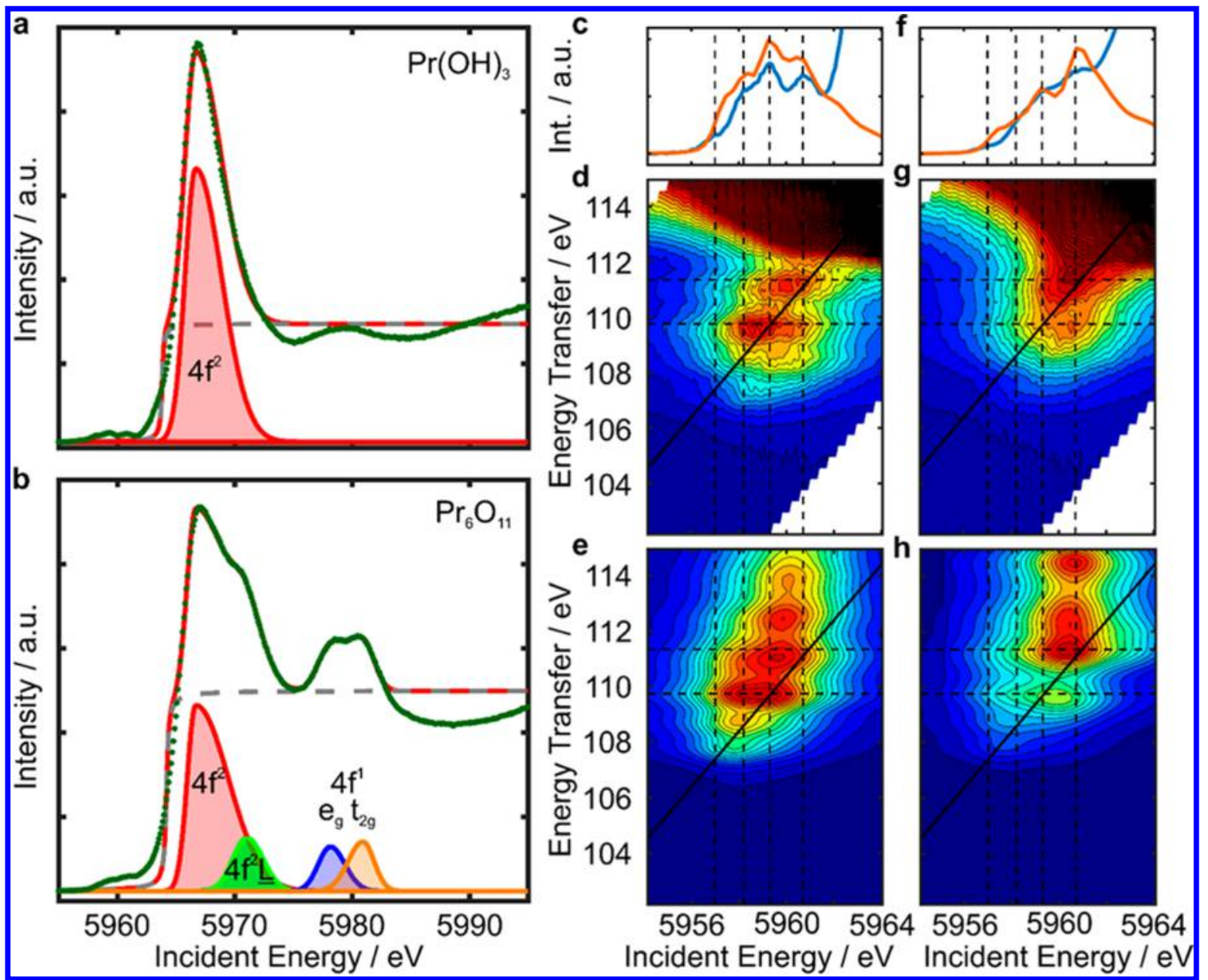

Figure 1. Experimental HERFD-XANES spectra $(\mathrm{a}, \mathrm{b})$ and experimental and calculated RIXS maps $(\mathrm{c}-\mathrm{h})$ of trivalent and mixed-valence praseodymium reference compounds. (a, b) Deconvolution of the HERFD-XANES spectra of the $\operatorname{Pr}(\mathrm{OH})_{3}$ and $\operatorname{Pr}_{6} \mathrm{O}_{11}$ nanoparticles, respectively. The $4 f^{2}$ (red), $4 f^{2} \underline{L}$ (green), and two $4 f^{1}$ features split by the crystal field (blue, orange) are fitted with SplitGaussians. The edge jump, fitted with an arctangent function, is shown as a gray dashed line. (c-e) Measured (d) and calculated (e) RIXS map for trivalent $\operatorname{Pr}(\mathrm{OH})_{3}$ showing four distinct features. ( $\mathrm{f}-\mathrm{h}$ ) Measured $(\mathrm{g})$ and calculated (h) RIXS maps of the mixed valence oxide $\operatorname{Pr}_{6} \mathrm{O}_{11}$. The pre-edge RIXS was calculated as a linear combination of $\mathrm{Pr}^{3+}$ and $\mathrm{Pr}^{4+}$. The dashed lines are guidelines for the eyes. The diagonal, bold, and dashed line indicates the fixed emission energy of $5849.63 \mathrm{eV}$, and the corresponding pre-edge spectra at this energy are extracted from the calculated maps (orange curve) and compared with the measured pre-edge spectra of $\operatorname{Pr}(\mathrm{OH})_{3}$ and $\operatorname{Pr}_{6} \mathrm{O}_{11}$ (blue curve) in (c) and (f).

pattern recorded with high resolution confirms the monoclinic $\mathrm{Pr}_{6} \mathrm{O}_{11}$ phase (Figures $\mathrm{S} 1$ and S2, Supporting Information).

TEM. Transmission electron microscopy measurements were conducted on a Philips Tecnai F30 operated at $300 \mathrm{kV}$ in the Scientific Center for Optical and Electron Microscopy (ScopeM) of the ETH Zurich. A Gatan 1k CCD chip was used for image acquisition via the software Digital Micrograph (Figure S3, Supporting Information).

XPS. X-ray photoelectron spectroscopy was recorded on a Sigma 2 spectrometer (Thermo Scientific) using a polychromatic $\mathrm{Al} \mathrm{K} \alpha \mathrm{X}$-ray source and the binding energy was calibrated taking C $1 \mathrm{~s}=284.4 \mathrm{eV}$ (Figure S4, Supporting Information).

TGA. Thermogravimetric analysis (TGA) was measured with a Mettler Toledo TGA/SDTA851e instrument. A total of $7.71 \mathrm{mg}$ of $\operatorname{Pr}(\mathrm{OH})_{3}$ powder was heated in an alumina crucible to $800{ }^{\circ} \mathrm{C}$ at a rate of $10^{\circ} \mathrm{C} / \mathrm{min}$ in air. After cooling down to $50{ }^{\circ} \mathrm{C}$ the powder was reheated to $350{ }^{\circ} \mathrm{C}$ at $10{ }^{\circ} \mathrm{C} / \mathrm{min}$ as shown in Figure S1b, Supporting Information.

Resistance Measurements. The $\operatorname{Pr}_{6} \mathrm{O}_{11}$ film was prepared on an alumina substrate, which was equipped with interdigitated platinum electrodes for the resistance measure- ments on the top side and with a meander type platinum heating element on the back side. A dispersion of $200 \mathrm{mg}$ of washed, but still wet, $\operatorname{Pr}(\mathrm{OH})_{3}$ nanoparticles mixed with $5 \mathrm{~mL}$ of ethanol was prepared. The substrate was heated to $75{ }^{\circ} \mathrm{C}$, and $10 \mu \mathrm{L}$ of the dispersion was drop cast 10 times on the substrate. Finally, the substrate with the film was heated to $500{ }^{\circ} \mathrm{C}$ for $2 \mathrm{~h}$ at a heating rate of $5{ }^{\circ} \mathrm{C} / \mathrm{min}$. Resistance was measured in a Teflon chamber under $200 \mathrm{~mL} / \mathrm{min}$ flow of dry synthetic air. The resistance of the film was measured with a Keithley 617 programmable electrometer, and the temperature was adjusted using a DC power supply.

\section{RESULTS}

The challenge in studying the mixed valence $\operatorname{Pr}_{6} \mathrm{O}_{11}$ is to unequivocally assign the spectral features related to $3+$ and $4+$ valence of $\operatorname{Pr}$. Thus, we study $\operatorname{Pr}(\mathrm{OH})_{3}$ as a trivalent reference and compare its spectral features with $\operatorname{Pr}_{6} \mathrm{O}_{11}$ as shown in Figure 1. We perform the experiments at the $\operatorname{Pr~L}_{3}$ edge $(2 p \rightarrow$ $5 d)$ absorption line and record the $\operatorname{Pr} \mathrm{L} \beta_{2,5}$ emission line $(4 d \rightarrow$ $2 p)$. The spectrometer setup in HERFD-XANES reduces the core-hole lifetime broadening from $3.6 \mathrm{eV}(2 p)^{22}$ to the $0.7 \mathrm{eV}$ 
$(4 d),{ }^{23-25}$ which is in the range of the experimental resolution of $1 \mathrm{eV}$. In the $\operatorname{Pr}(\mathrm{OH})_{3}$ spectrum, we assign the single feature to the $4 f^{2} 5 d^{1}$ state as shown in Figure 1a. The $4 f^{3} \underline{L} 5 d^{1}$ state, where $\underline{L}$ denotes a hole in the ligand $\mathrm{O} 2 p$ orbital, is not visible due to broadening of $\operatorname{Pr} 5 d$ states related to oxygen as neighboring atoms. ${ }^{26}$ In the spectrum of $\operatorname{Pr}_{6} \mathrm{O}_{11}$, additionally to the $4 f^{2} 5 d^{1}$ final state characteristic for $\operatorname{Pr}^{3+}$, we observe three more features, which manifest the presence of $\mathrm{Pr}^{4+}$ as shown in Figure $1 \mathrm{~b}$. The quasi-cubic like surrounding of the Pr ions in the monoclinic $\operatorname{Pr}_{6} \mathrm{O}_{11}$ results in a cubic crystal field splitting of the states into an $e_{g}$ and $t_{2 g}$ state with an energy difference of $2.8 \mathrm{eV}^{27}$ Thus, by analogy to $\mathrm{CeO}_{2}$ and $\mathrm{PrO}_{2}$, we assign the features at $5978.2 \mathrm{eV}$ (blue) and $5980.9 \mathrm{eV}$ (orange) to the $4 f^{1} 5 d^{1}$ final state. ${ }^{18,19,28,29}$ The $t_{2 g}$ final state of $4 f^{2} \underline{L} 5 d^{1}$ appears at $5971.0 \mathrm{eV},{ }^{18}$ whereas the $\mathrm{e}_{\mathrm{g}}$ peak overlaps with $4 f^{2} 5 d^{1}$ of $\operatorname{Pr}^{3+}$.

From a theoretical point of view, it is challenging to treat the partly filled $4 f$ orbitals of the $\mathrm{RE}$ ions. In the sesquioxides $\mathrm{RE}_{2} \mathrm{O}_{3}$, the $\mathrm{RE}$ donates three electrons from the outer shells to the bond and the remaining $4 f$ electrons stay strongly localized at the trivalent metal ion. ${ }^{13}$ For $\mathrm{Ce}, \mathrm{Tb}$, and $\mathrm{Pr}$ also tetravalent ions exist, and due to the hybridization with the $\mathrm{O} 2 p$ states the $f$-electron occupancy takes a noninteger value, which results in a certain degree of delocalization. Here, $\operatorname{Pr}^{3+}$ represents a $4 f^{2}$ configuration and $\operatorname{Pr}^{4+}$ a $4 f^{1}$ and $4 f^{2} \underline{L}$ configuration, which includes a delocalization of the $4 f$ states. The relation between those well-resolved features allows determining the $f$-electron occupancy $\left(n_{f}\right)$ and the formal valence $(v)$ with high accuracy. Here, the $\mathrm{Pr}^{4+} / \mathrm{Pr}^{3+}$ ratio is $0.53 \pm 0.13$ and valence $v=3.53 \pm$ $0.13,{ }^{30}$ which is close to the expected value of 3.66 for $\operatorname{Pr}_{6} \mathrm{O}_{11}$. The $n_{f}$ of $\operatorname{Pr}_{6} \mathrm{O}_{11}$ is $1.79 \pm 0.04$, which is as expected higher than 1.65 determined with conventional XANES for the stoichiometric $\mathrm{PrO}_{2}{ }^{31}$

Furthermore, in the pre-edge region we observe strong differences between $\operatorname{Pr}(\mathrm{OH})_{3}$ and $\operatorname{Pr}_{6} \mathrm{O}_{11}$. We record the RIXS maps because the quadrupole transitions from $2 p \rightarrow 4 f$ states are spread out differently in the incident beam and energy transfer directions depending on the number of localized $f$ electrons, shown in Figure $1 \mathrm{c}-\mathrm{h} .{ }^{11}$ In the RIXS map calculations, we do not take into account dipole $2 p \rightarrow 5 d$ transitions and mixing of the $5 d$ states with the $4 f^{2}$ states, but instead solely focus on the process $2 p \rightarrow 4 f$ and $4 d \rightarrow 2 p$. For $\operatorname{Pr}(\mathrm{OH})_{3}$, the calculated RIXS maps reproduce very well all four features with the correct broadening in incident energy and energy transfer (Figure $1 \mathrm{c}-\mathrm{e}$ ). In the $\mathrm{Pr}_{6} \mathrm{O}_{11}$ RIXS maps, the four features distinguished in the $\operatorname{Pr}(\mathrm{OH})_{3}$ sample are clearly visible but have different relative intensities due to the presence of tetravalent $\operatorname{Pr}$ ions (Figure $1 \mathrm{f}-\mathrm{h}$ ). We calculate the $\operatorname{Pr}_{6} \mathrm{O}_{11}$ maps as a linear combination of $\operatorname{Pr}^{3+}$ (Figure 1e) and $\operatorname{Pr}^{4+}$ (Figure S5, Supporting Information) maps and take two trivalent and four tetravalent ions into account. The relative intensities of the features in the calculations and the measured intensities are in the right order of magnitude, while the calculation underestimates the broadening.

We study the emergence of $\operatorname{Pr}^{4+}$ by monitoring the $f$-electron occupancy $\left(n_{f}\right)$ and the normalized area below the pre-edge $\left(n_{A}\right)$ during the temperature-induced phase-transformation from $\operatorname{Pr}(\mathrm{OH})_{3}$ to $\operatorname{Pr}_{6} \mathrm{O}_{11}$, as shown in Figure 2 and Figure $\mathrm{S} 1$, Supporting Information. At $300{ }^{\circ} \mathrm{C}$, a decrease of $n_{f}$ and an increase of $n_{A}$ sets in and reaches a plateau above $500{ }^{\circ} \mathrm{C}$. We assign these changes to the formation of $\mathrm{Pr}^{4+}$, which is in line with the phase transformation from $\operatorname{Pr}(\mathrm{OH})_{3}$ to $\operatorname{Pr}_{6} \mathrm{O}_{11}$ observed in PXRD and TGA studies. Surprisingly, upon

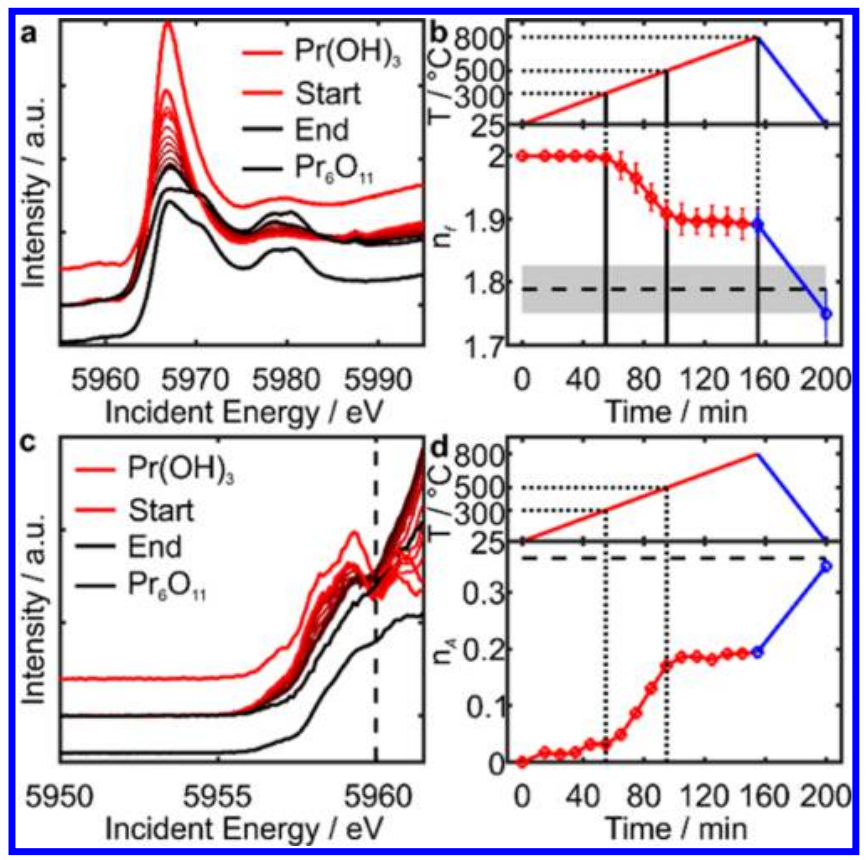

Figure 2. In situ HERFD-XANES studies of the temperature-induced phase transition from $\operatorname{Pr}(\mathrm{OH})_{3}$ to $\operatorname{Pr}_{6} \mathrm{O}_{11}$. The reference spectra of $\operatorname{Pr}(\mathrm{OH})_{3}$ and $\operatorname{Pr}_{6} \mathrm{O}_{11}$ measured at RT are down-/up-shifted, respectively. ( $\mathrm{a}, \mathrm{b})$ HERFD-XANES spectra measured at RT and then every $50{ }^{\circ} \mathrm{C}$ from 50 to $800{ }^{\circ} \mathrm{C}$ and the corresponding $f$-electron occupancy $n_{f}$ as a function of time and temperature. As expected, the formation of $\operatorname{Pr}^{4+}$ ions at $300{ }^{\circ} \mathrm{C}$ leads to the decrease of the $f$-electron occupancy. Additionally, the $n_{f}$ strongly decreases toward RT and reaches the ex situ determined value of $1.79 \pm 0.04$ (gray area). This demonstrates the changing occupancy of the $\mathrm{O} 2 p$-Pr $4 f$ band with temperature. (c, d) Pre-edge spectra and the corresponding normalized pre-edge area, $n_{A}$, confirm the formation of $\operatorname{Pr}_{6} \mathrm{O}_{11}$. The increase of $n_{A}$ together with the shape conservation of the pre-edge at RT after cooling down supports the higher delocalization at high temperatures rather than an oxidation of $\mathrm{Pr}^{3+}$ to $\mathrm{Pr}^{4+}$ upon cooling.

cooling from $800{ }^{\circ} \mathrm{C}$ to room temperature (RT), $n_{f}$ and $n_{A}$ further strongly change to finally reach the values measured for the reference compound at RT. The possible explanation for the sharp decrease of the $n_{f}$ and increase of $n_{A}$ upon cooling can be either a further oxidation of $\operatorname{Pr}^{3+}$ to $\operatorname{Pr}^{4+}$ or temperatureinduced changes of the occupancy of the $\mathrm{O} 2 p$-Pr $4 f$ band. As shown in Figure S6, Supporting Information, the mass loss between 800 and $50{ }^{\circ} \mathrm{C}$ is only $0.5 \%$ and thus we can discard the hypothesis of oxidation of $\mathrm{Pr}^{3+}$ to $\mathrm{Pr}^{4+}$. An inspection of the spectra measured at $800{ }^{\circ} \mathrm{C}$ and RT reveals that in both cases we observe all the postedge features of $\operatorname{Pr}_{6} \mathrm{O}_{11}$, but at RT the white line maximum is flat. In the pre-edge, the two features at 5958.2 and $5959.3 \mathrm{eV}$ are characteristic for the $4 f^{2}$ state (Figure $1 \mathrm{c}-\mathrm{e})$. For the measurements at $800{ }^{\circ} \mathrm{C}$ and $\mathrm{RT}$, the relative intensities of those shoulders are very similar. However, at RT all the pre-edge features are generally shifted to higher energies. This finding supports the assumption of a higher delocalization at elevated temperatures rather than a shift from $4 f^{1}$ to $4 f^{2}$ or vice versa. The shift of the pre-edge to lower energies with a higher degree of $f$-state delocalization was observed for $\mathrm{CeO}_{2}$ nanoparticles. ${ }^{8}$ Furthermore, for the transition metals a temperature dependency of the quadrupole transitions due to the additional vibrational modes was reported. ${ }^{32,33}$

To further test our hypothesis we heat $\operatorname{Pr}_{6} \mathrm{O}_{11}$ nanopowders again from RT to $350{ }^{\circ} \mathrm{C}$ and compare the spectroscopic changes $\left(n_{f}, n_{A}\right)$ with the resistance measurements of the $\operatorname{Pr}_{6} \mathrm{O}_{11}$ 


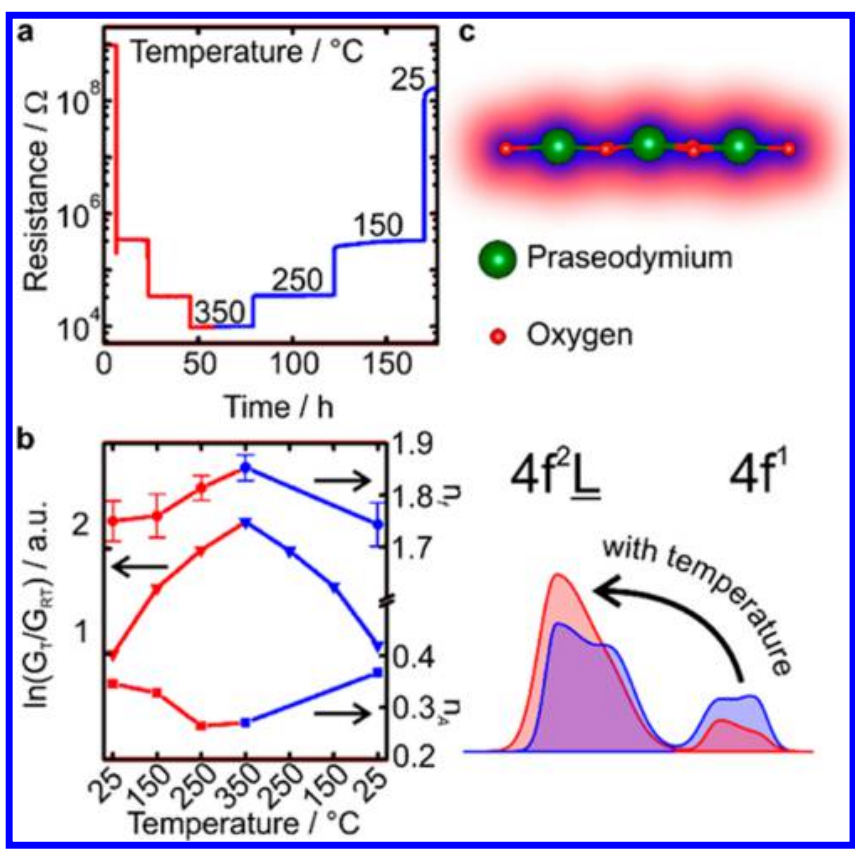

Figure 3. Reversible changes of the f-electron occupancy in $\operatorname{Pr}_{6} \mathrm{O}_{11}$ upon heating and their correlation with the electrical conductance. (a) Temperature-induced changes of resistance of $\operatorname{Pr}_{6} \mathrm{O}_{11}$ films. (b) Dependence of the conductance, $\ln \left(G_{n} / G_{\mathrm{RT}}\right)$, to the temperature during heating and cooling (left axis). The reversible changes of the conductance with temperature illustrate the effect of the electron delocalization on this macroscopic property. Both the analysis of the $f$ electron occupancy, $n_{f}$ (top right axis), and the normalized pre-edge area, $n_{A}$ (bottom right axis), show the higher occupancy of the $\mathrm{O} 2 p$-Pr $4 f$ band at higher temperature. (c) Model of O $2 p-\operatorname{Pr} 4 f$ hybridization is shown. The $4 f^{2} \underline{\mathrm{L}}$ feature increases with temperature resulting in higher $f$-electron occupancy. (top) Artistic view on the delocalization of electrons at different temperatures and (bottom) the corresponding HERFD-XANES spectra. The blue and red colors refer to the low and high temperature, respectively.

films $\left(\ln \left(G / G_{\mathrm{RT}}\right)\right)$ as shown in Figure 3a,b and Figure S7a,b, Supporting Information. We determine the electronic band gap of the $\mathrm{Pr}_{6} \mathrm{O}_{11}$ nanoparticles from valence-to-core X-ray emission spectroscopy (vtc XES) and HERFD-XANES to be $2.04 \mathrm{eV}$ as shown in Figure S8, Supporting Information. As expected for an n-type semiconductor, the resistance $R_{\mathrm{n}}$ of the $\operatorname{Pr}_{6} \mathrm{O}_{11}$ film decreases by 5 orders of magnitude upon heating to $350{ }^{\circ} \mathrm{C}$ and recovers to the almost initial value after cooling. This can be explained by the dependency of the delocalized charge carrier concentration on temperature, which is proportional to $\exp \left(-E / k_{\mathrm{B}} T\right)$, where $E$ is the energy needed to excite electrons into the conduction band. In this temperature range, we can exclude any phase transition (see Figure S7c,d, Supporting Information), but we still observe reversible changes of $n_{f}$ and $n_{A}$ upon cooling and heating. We compile all the information extracted from the HERFD-XANES studies and resistance measurements to visualize how the temperatureinduced delocalization of $f$-electrons affects the spectral features as shown in Figure 3c.

\section{CONCLUSION}

In summary, the electrical measurements are in line with the findings based on X-ray spectroscopy. Upon heating, we observe a higher degree of delocalization of the $f$-electrons and therefore a higher electrical conductivity. The analysis of HERFD-XANES spectra and RIXS maps allows us to unambiguously distinguish between the temperature-induced changes of the Pr valence state from the changes exclusively related to delocalization of the $f$-electrons. Therefore, the photon-in photon-out method can be used as a contactless probe of the delocalized electrons in the conduction band of semiconductors.

As worldwide high brilliance sources and emission spectrometers are under rapid development, we anticipate that this work will trigger a further application of this method to monitor electrical properties of semiconducting nanomaterials in operando conditions and will be particularly interesting in the field of thermo-electrics.

\section{ASSOCIATED CONTENT}

\section{Supporting Information}

The Supporting Information is available free of charge on the ACS Publications website at DOI: 10.1021/acs.chemmater.6b05218.

Electronic band gap determination, RIXS calculations for $\mathrm{Pr}^{4+}$, TEM images, XPS data, in situ PXRD and TGA data, reheated HERFD-XANES, PXRD, and TGA data, and HR-PXRD of $\operatorname{Pr}(\mathrm{OH})_{3}$ and $\mathrm{Pr}_{6} \mathrm{O}_{11}$. The reduced Slater integrals for the multiplet calculations are listed. (PDF)

\section{AUTHOR INFORMATION}

\section{Corresponding Author}

*(D.K.) E-mail: dorota.koziej@mat.ethz.ch.

ORCID

Dorota Koziej: 0000-0002-9064-2642

Notes

The authors declare no competing financial interest.

\section{ACKNOWLEDGMENTS}

We thank the Eidgenössische Technische Hochschule Zürich (ETH 2813-1) for financial support and the European Synchrotron Radiation Facility for beamtime allocation at ID26 and Swiss-Norwegian Beamlines BM01A. We thank Hugo Vittoux for the assistance with the furnace and Dr. Dmitry Chernyshov for the help with the interpretation of highresolution PXRD data. We acknowledge Dr. Elena TervoortGorokhova for TEM measurements and Dr. Guobo Zeng for XPS measurements.

\section{REFERENCES}

(1) Snyder, G. J.; Toberer, E. S. Complex thermoelectric materials. Nat. Mater. 2008, 7, 105-114.

(2) Friebel, D.; Louie, M. W.; Bajdich, M.; Sanwald, K. E.; Cai, Y.; Wise, A. M.; Cheng, M.-J.; Sokaras, D.; Weng, T.-C.; Alonso-Mori, R.; Davis, R. C.; Bargar, J. R.; Nørskov, J. K.; Nilsson, A.; Bell, A. T. Identification of Highly Active $\mathrm{Fe}$ Sites in $(\mathrm{Ni}, \mathrm{Fe}) \mathrm{OOH}$ for Electrocatalytic Water Splitting. I. Am. Chem. Soc. 2015, 137, 13051313.

(3) Franklin, A. D. Nanomaterials in transistors: From highperformance to thin-film applications. Science 2015, 349, aab2750.

(4) Fiori, G.; Bonaccorso, F.; Iannaccone, G.; Palacios, T.; Neumaier, D.; Seabaugh, A.; Banerjee, S. K.; Colombo, L. Electronics based on two-dimensional materials. Nat. Nanotechnol. 2014, 9, 768-779.

(5) Radisavljevic, B.; Kis, A. Mobility engineering and a metalinsulator transition in monolayer $\mathrm{MoS}_{2}$. Nat. Mater. 2013, 12, 815820.

(6) Zheng, H.; Rivest, J. B.; Miller, T. A.; Sadtler, B.; Lindenberg, A.; Toney, M. F.; Wang, L.-W.; Kisielowski, C.; Alivisatos, A. P. 
Observation of transient structural-transformation dynamics in a $\mathrm{Cu}_{2} \mathrm{~S}$ danorod. Science 2011, 333, 206-209.

(7) Hirsch, O.; Kvashnina, K. O.; Luo, L.; Süess, M. J.; Glatzel, P.; Koziej, D. High-energy resolution $\mathrm{X}$-ray absorption and emission spectroscopy reveals insight into unique selectivity of La-based nanoparticles for $\mathrm{CO}_{2}$. Proc. Natl. Acad. Sci. U. S. A. 2015, 112, 15803-15808.

(8) Cafun, J.-D.; Kvashnina, K. O.; Casals, E.; Puntes, V. F.; Glatzel, P. Absence of $\mathrm{Ce}^{3+}$ sites in chemically active colloidal ceria nanoparticles. ACS Nano 2013, 7, 10726-10732.

(9) Safonova, O. V.; Guda, A. A.; Paun, C.; Smolentsev, N.; Abdala, P. M.; Smolentsev, G.; Nachtegaal, M.; Szlachetko, J.; Soldatov, M. A.; Soldatov, A. V.; van Bokhoven, J. A. Electronic and Geometric Structure of $\mathrm{Ce}^{3+}$ Forming Under Reducing Conditions in Shaped Ceria Nanoparticles Promoted by Platinum. I. Phvs. Chem. C 2014, 118, 1974-1982.

(10) Koziej, D. Revealing Complexity of Nanoparticle Synthesis in Solution by in Situ Hard X-ray Spectroscopy-Today and Beyond. Chem. Mater. 2016, 28, 2478-2490.

(11) Kvashnina, K. O.; Butorin, S. M.; Glatzel, P. Direct study of the $f$-electron configuration in lanthanide systems. I. Anal. At. Spectrom. 2011, 26, 1265-1272.

(12) Rao, G. V. S.; Ramdas, S.; Mehrotra, P. N.; Rao, C. N. R. Electrical transport in rare-earth oxides. I. Solid State Chem. 1970, 2, 377-384.

(13) Petit, L.; Svane, A.; Szotek, Z.; Temmerman, W. M. Firstprinciples study of rare-earth oxides. Phvs. Rev. B: Condens. Matter Mater. Phvs. 2005, 72, 205118.

(14) Butler, P. H. Point Group Symmetry and Applications - Methods and Tables; Plenum Press: New York, 1981.

(15) Cowan, R. D. The Theory of Atomic Structure and Spectra; University of California Press: Berkeley, CA, 1981.

(16) Kotani, A.; Shin, S. Resonant inelastic x-ray scattering spectra for electrons in solids. Rev. Mod. Phvs. 2001, 73, 203-246.

(17) Wojdyr, M. Fityk: a general-purpose peak fitting program. $I$. Appl. Crustallogr. 2010, 43, 1126-1128.

(18) Bianconi, A.; Kotani, A.; Okada, K.; Giorgi, R.; Gargano, A.; Marcelli, A.; Miyahara, T. Many-body effects in praesodymium corelevel spectroscopies of $\mathrm{PrO}_{2}$. Phvs. Rev. B: Condens. Matter Mater. Phvs. 1988, 38, 3433-3437.

(19) Bianconi, A.; Marcelli, A.; Dexpert, H.; Karnatak, R.; Kotani, A.; Jo, T.; Petiau, J. Specific intermediate-valence state of insulating $4 f$ compounds detected by $\mathrm{L}_{3} \mathrm{X}$-ray absorption. Phvs. Rev. B: Condens. Matter Mater. Phys. 1987, 35, 806-812.

(20) Young, R. A. The Rietveld Method; Oxford University Press: 1993.

(21) Rodríguez-Carvajal, J. Recent advances in magnetic structure determination by neutron powder diffraction. Phvs. B 1993, 192, 5569.

(22) Krause, M. O.; Oliver, J. H. Natural widths of atomic K and L levels, K $\alpha$ X-ray lines and several KLL Auger lines. I.Phys. Chem. Ref. Data 1979, 8, 329-338.

(23) Tanaka, S.; Ogasawara, H.; Okada, K.; Kotani, A. Theory of the $4 \mathrm{~d} \rightarrow 2 \mathrm{p}$ X-ray emission spectroscopy in $\mathrm{Ce}_{2} \mathrm{O}_{3}, \mathrm{Pr}_{2} \mathrm{O}_{3}$ and $\mathrm{Dy}_{2} \mathrm{O}_{3}$. I. Phvs. Soc. Ipn. 1995, 64, 2225-2232.

(24) Ogasawara, H.; Kotani, A.; Thole, B. T. Lifetime effect on the multiplet structure of $4 d \mathrm{x}$-ray-photoemission spectra in heavy rareearth elements. Phys. Rev. B: Condens. Matter Mater. Phys. 1994, 50, 12332-12341.

(25) Ogasawara, H.; Kotani, A.; Potze, R.; Sawatzky, G. A.; Thole, B. T. Praseodymium $3 d$ - and $4 d$-core photoemission spectra of $\operatorname{Pr}_{2} \mathrm{O}_{3}$. Phvs. Rev. B: Condens. Matter Mater. Phvs. 1991, 44, 5465-5469.

(26) Yamaoka, H.; Sugiyama, H.; Kubozono, Y.; Kotani, A.; Nouchi, R.; Vlaicu, A. M.; Oohashi, H.; Tochio, T.; Ito, Y.; Yoshikawa, H. Charge transfer satellite in $\operatorname{Pr} @ \mathrm{C}_{82}$ metallofullerene observed using resonant x-ray emission spectroscopy. Phys. Rev. B: Condens. Matter Mater. Phvs. 2009, 80, 205403.

(27) Kotani, A.; Kvashnina, K. O.; Butorin, S. M.; Glatzel, P. Spectator and participator processes in the resonant photon-in and photon-out spectra at the $\mathrm{Ce}_{3}$ edge of $\mathrm{CeO}_{2}$. Eur. Phys. I. B 2012, 85, 257.

(28) Karnatak, R. C.; Esteva, J. M.; Dexpert, H.; Gasgnier, M.; Caro, P. E.; Albert, L. X-ray absorption studies of $\mathrm{CeO}_{2}, \mathrm{PrO}_{2}$, and $\mathrm{TbO}_{2}$. I. Manifestation of localized and extended $f$ states in the $3 d$ absorption spectra. Phvs. Rev. B: Condens. Matter Mater. Phys. 1987, 36, 17451749.

(29) Dexpert, H.; Karnatak, R. C.; Esteva, J. M.; Connerade, J. P.; Gasgnier, M.; Caro, P. E.; Albert, L. X-ray absorption studies of $\mathrm{CeO}_{2}$, $\mathrm{PrO}_{2}$, and $\mathrm{TbO}_{2}$. II. Rare-earth valence state by $\mathrm{L}_{\mathrm{III}}$ absorption edges. Phvs. Rev. B: Condens. Matter Mater. Phvs. 1987, 36, 1750-1753.

(30) Yamaoka, H.; Oohashi, H.; Jarrige, I.; Terashima, T.; Zou, Y.; Mizota, H.; Sakakura, S.; Tochio, T.; Ito, Y.; Sherman, E. Y.; Kotani, A. $\mathrm{X}$-ray spectroscopic study of the electronic structure of $\mathrm{Y}_{1-\mathrm{x}} \mathrm{Pr}_{\mathrm{x}} \mathrm{Ba}_{2} \mathrm{Cu}_{3} \mathrm{O}_{7}$. Phvs. Rev. B: Condens. Matter Mater. Phys. 2008, 77,045135

(31) Tran, F.; Schweifer, J.; Blaha, P.; Schwarz, K.; Novák, P. PBE+U calculations of the Jahn-Teller effect in $\mathrm{PrO}_{2}$. Phys. Rev. B: Condens. Matter Mater. Phvs. 2008, 77, 085123.

(32) Durmeyer, O.; Beaurepaire, E.; Kappler, J. P.; Brouder, Ch.; Baudelet, $\mathrm{F}$. Temperature dependence of the pre-edge structure in the Ti K-edge X-ray absorption spectrum of rutile. I. Phvs.: Condens. Matter 2010, 22, 125504.

(33) Brouder, C.; Cabaret, D.; Juhin, A.; Sainctavit, P. Effect of atomic vibrations on the $\mathrm{X}$-ray absorption spectra at the $\mathrm{K}$ edge of $\mathrm{Al}$ in $\alpha-\mathrm{Al}_{2} \mathrm{O}_{3}$ and of $\mathrm{Ti}$ in $\mathrm{TiO}_{2}$ rutile. Phys. Rev. B: Condens. Matter Mater. Phvs. 2010, 81, 115125. 